\title{
On the Best Constant Problem for a Class of Mixed-Norm Hardy Inequalities
}

\author{
Baofeng Lai ${ }^{1} \cdot$ Runqiu Wang ${ }^{2}$
}

Received: 18 October 2020 / Accepted: 6 March 2021 / Published online: 22 March 2021

(C) The Author(s) 2021

\begin{abstract}
In this paper, we obtain the best constant and the equality condition for a class of mixed-norm Hardy inequalities when the weight is a power function. By building and solving the corresponding Euler equation, we look for the best constant and the optimal function. One of the main ingredients is to introduce two key auxiliary functions so that the corresponding equalities are derived.
\end{abstract}

Keywords Hardy inequality $\cdot$ Mixed-norm $\cdot$ Best constant $\cdot$ Equality condition

\section{Introduction}

If the powers of the integrands on both sides of the Hardy inequality are different, we call this kind of Hardy inequality as the mixed-norm Hardy inequality. The resulting method, best constant and condition of equality of the mixed-norm Hardy inequality are rather different from the ones for the usual Hardy inequality.

By our knowledge, the earliest example of the mixed-norm Hardy inequality was given in 1930. In the paper [1], G.H. Hardy and J.E. Littlewood presented a conjecture of the following inequality by adopting the variational method and solving the corresponding Euler equation:

Let $l>k>1, r=\frac{l}{k}-1, f$ is a non-negative and measurable function in $(0, \infty)$ and $0<\int_{0}^{\infty} f^{k}(x) d x<\infty$. Let $F(x)=\int_{0}^{x} f(t) d t$, then

$$
\int_{0}^{\infty} x^{r-l} F^{l}(x) d x \leq K\left[\int_{0}^{\infty} f^{k}(x) d x\right]^{\frac{l}{k}},
$$

B. Lai

laibaofeng@sina.cn

1 Department of Mathematics, Nanjing University, Nanjing, Jiangsu, 210093, China

2 School of Mathematical Sciences, Jiangsu University, Zhenjiang, Jiangsu, 212013, China 
where $K=\frac{1}{l-r-1}\left[\frac{r \Gamma\left(\frac{l}{r}\right)}{\Gamma\left(\frac{1}{r}\right) \Gamma\left(\frac{l-1}{r}\right)}\right]^{r}$ is the best constant, and the equality holds if and only if $F(x)=\frac{x}{\left(\alpha x^{r}+\beta\right)^{\frac{1}{r}}}(\alpha, \beta>0)$.

It is noted that the strict proof of the inequality (1.1) was first given by G.A. Bliss in [2]. In 1958, T.M. FLett gave another mixed-norm Hardy inequality in [3]:

Let $f(t) \geq 0$ in $t \geq 0$, and let $F_{\alpha}(t)$ be the $\alpha$-th Riemann-Liouville integral of $f$ with origin 0 , i.e.

$$
F_{\alpha}(t)=\frac{1}{\Gamma(\alpha)} \int_{0}^{t}(t-u)^{\alpha-1} f(u) d u .
$$

If either $q \geq p \geq 1$ and $\alpha>\frac{1}{p}-\frac{1}{q}$, or $q>p>1$ and $\alpha=\frac{1}{p}-\frac{1}{q}$, and if $\gamma>-1$, then there exists a positive constant $B$, such that

$$
\left\{\int_{0}^{\infty} t^{-1-q \gamma}\left(\frac{F_{\alpha}}{t^{\alpha}}\right)^{q} d t\right\}^{\frac{1}{q}} \leq B\left\{\int_{0}^{\infty} t^{-1-p \gamma} f^{p} d t\right\}^{\frac{1}{p}} .
$$

When $q>p, \alpha=1-\frac{1}{q}, \gamma=-\frac{1}{p}$ and $p^{\prime}>q$, then

$$
B=\frac{\pi^{\frac{1}{p^{\prime}}}}{\Gamma\left(1-\frac{1}{q}\right)}\left\{\frac{\Gamma^{2}\left(\frac{q}{2 p^{\prime}}\right)}{\Gamma\left(\frac{q}{p^{\prime}}\right)}\right\}^{\frac{1}{q}}=\frac{\pi^{\frac{1}{p^{\prime}}}}{\Gamma\left(1-\frac{1}{q}\right)}\left\{\frac{\pi^{\frac{1}{2}} \Gamma\left(\frac{q}{2 p^{\prime}}\right)}{2^{\frac{q}{p^{\prime}}-1} \Gamma\left(\frac{q}{2 p^{\prime}}+\frac{1}{2}\right)}\right\}^{\frac{1}{q}} .
$$

When $q=p>1$, then $B=\frac{\Gamma(\alpha) \Gamma(\gamma+1)}{\Gamma(\alpha+\gamma+1)}$.

The Young inequality for convolution operator is a suitable tool to deal with the mixednorm Hardy inequality. However, this method can't give the best constant and the condition of equality in general. In the monograph [4] on the integral inequalities, Claude George proved the following inequality by using the Young inequality for convolution operator:

Suppose that $p \geq 1, q \geq 1, r \geq 1, \frac{1}{p}+\frac{1}{q}=1, \frac{1}{p}+\frac{1}{r}-1>0, f$ is non-negative and measurable in $(0, \infty)$ and satisfies $\int_{0}^{\infty} f^{p}(x) d x<\infty$. Let $\frac{1}{\alpha}=\frac{1}{p}+\frac{1}{r}-1, \beta=\frac{1}{\alpha}+\frac{1}{q}$, $F(x)=\frac{1}{x} \int_{0}^{x} f(t) d t$, then

$$
\left\{\int_{0}^{\infty} x^{\frac{\alpha}{p}-1} F^{\alpha}(x) d x\right\}^{\frac{1}{\alpha}} \leq\left(\frac{q}{\alpha}+1\right)^{\beta}\|f\|_{p} .
$$

In [5], J.S. Bradley proved the following general conclusions about the weighted mixednorm Hardy inequality, which are the generalizations of the case $p=q \geq 1$ in [6]:

Theorem 1.1 Suppose that $1 \leq p, q \leq \infty, p^{\prime}$ is the conjugate number of $p, u(x), v(x) \geq 0$.

(a) Let $\int_{0}^{r} v^{-p^{\prime}}(x) d x<\infty$ for all $0<r<\infty$. If there exists a constant $C>0$, such that

$$
\left\{\int_{0}^{\infty}\left[u(x) \int_{0}^{x} f(t) d t\right]^{q} d x\right\}^{\frac{1}{q}} \leq C\left\{\int_{0}^{\infty}[v(x) f(x)]^{p} d x\right\}^{\frac{1}{p}}
$$

holds for all admissible $f \geq 0$, then

$$
B=\sup _{r>0}\left[\int_{r}^{\infty} u^{q}(x) d x\right]^{\frac{1}{q}}\left[\int_{0}^{r} v^{-p^{\prime}}(x) d x\right]^{\frac{1}{p^{\prime}}}<\infty .
$$

Moreover, in case $p \leq q$, then (1.3) is also sufficient for (1.2) with all admissible $f$. 
(b) Let $\int_{r}^{\infty} v^{-p^{\prime}}(x) d x<\infty$ for all $0<r<\infty$, then the existence of a constant $C>0$ such that

$$
\left\{\int_{0}^{\infty}\left[u(x) \int_{x}^{\infty} f(t) d t\right]^{q} d x\right\}^{\frac{1}{q}} \leq C\left\{\int_{0}^{\infty}[v(x) f(x)]^{p} d x\right\}^{\frac{1}{p}}
$$

holds for all admissible $f$ implies that

$$
B=\sup _{r>0}\left[\int_{0}^{r} u^{q}(x) d x\right]^{\frac{1}{q}}\left[\int_{r}^{\infty} v^{-p^{\prime}}(x) d x\right]^{\frac{1}{p^{\prime}}}<\infty .
$$

When $p \leq q$, (1.5) is also sufficient for (1.4), to hold for all admissible $f$.

Finally, in all cases, if $C$ denotes the best constant in (1.2) or (1.4), then $B \leq C \leq$ $B p^{\frac{1}{q}}\left(p^{\prime}\right)^{\frac{1}{p^{\prime}}}$ for $1<p \leq q<\infty$, while $C=B$, if $p=1$ or $q=\infty$.

In [7], P.R. Beesack and H.P. Heinig gave the following two dual conclusions including the negative exponent.

Theorem 1.2 Suppose that $p, q<0, p^{\prime}=\frac{p}{p-1}$ is the conjugate number of $p, u(x), v(x) \geq 0$.

(a) Let $0<\int_{0}^{r} v^{-p^{\prime}}(x) d x<\infty$ for all $r>0$. If there exists $C>0$ such that

$$
\left\{\int_{0}^{\infty}\left[u(x) \int_{0}^{x} f(t) d t\right]^{q} d x\right\}^{\frac{1}{q}} \geq C\left\{\int_{0}^{\infty}[v(x) f(x)]^{p} d x\right\}^{\frac{1}{p}}
$$

holds for all admissible $f$, then

$$
\inf _{r>0} K(r)=\inf _{r>0}\left[\int_{0}^{r} u^{q}(x) d x\right]^{\frac{1}{q}}\left[\int_{0}^{r} v^{-p^{\prime}}(x) d x\right]^{\frac{1}{p^{\prime}}}=B>0 .
$$

Moreover, in case $q \leq p<0, K(r)$ not only satisfies (1.7) but also is non-decreasing on $R^{+}$, then (1.6) holds for some $C>0$ and all admissible $f$.

(b) If $p, q<0,0<\int_{r}^{\infty} v^{-p^{\prime}}(x) d x<\infty$ for all $r>0$. If there exists some $C>0$, such that the following inequality

$$
\left\{\int_{0}^{\infty}\left[u(x) \int_{x}^{\infty} f(t) d t\right]^{q} d x\right\}^{\frac{1}{q}} \geq C \int_{0}^{\infty}[v(x) f(x)]^{p} d x
$$

holds for all admissible $f$, then

$$
\inf _{r>0} J(r)=\inf _{r>0}\left[\int_{r}^{\infty} u^{q}(x) d x\right]^{\frac{1}{q}}\left[\int_{r}^{\infty} v^{-p^{\prime}}(x) d x\right]^{\frac{1}{p^{\prime}}}=B>0 .
$$

Moreover, in case $q \leq p<0, J(r)$ not only satisfies (1.9) but also is non-increasing on $R^{+}$, then (1.8) holds for some $C>0$ and all admissible $f$.

As in Theorem 1.1, the best constant $C$ in (1.6) and (1.8) satisfies $B \geq C \geq|p|^{\frac{1}{q}}\left(p^{\prime}\right)^{\frac{1}{p^{\prime}}} B$, if $q \leq p<0$. 
In [7], P.R. Beesack and H.P. Heinig also gave the following two dual conclusions when the exponents $p, q \in(0,1)$.

Theorem 1.3 Suppose that $0<p, q<1, p^{\prime}=\frac{p}{p-1}$ is the conjugate number of $p$, $u(x), v(x) \geq 0$.

(a) Let $0<\int_{r}^{\infty} u^{q}(x) d x, \int_{r}^{\infty} v^{-p^{\prime}}(x) d x<\infty$ for all $r>0$. If there exists $C>0$ such that

$$
\left\{\int_{0}^{\infty}\left[u(x) \int_{0}^{x} f(t) d t\right]^{q} d x\right\}^{\frac{1}{q}} \geq C\left\{\int_{0}^{\infty}[v(x) f(x)]^{p} d x\right\}^{\frac{1}{p}}
$$

holds for all admissible $f$, then

$$
\inf _{r>0} J(r)=\inf _{r>0}\left[\int_{r}^{\infty} u^{q}(x) d x\right]^{\frac{1}{q}}\left[\int_{r}^{\infty} v^{-p^{\prime}}(x) d x\right]^{\frac{1}{p^{\prime}}}=B>0 .
$$

Moreover if $0<q \leq p<1, J(r)$ not only satisfies (1.11) but also is non-increasing, then (1.10) holds for all admissible $f$ with $B \geq C \geq p^{\frac{1}{q}}\left|p^{\prime}\right|^{\frac{1}{p^{\prime}}} B$.

(b) Let $0<\int_{0}^{r} u^{q}(x) d x, \int_{0}^{r} v^{-p^{\prime}}(x) d x<\infty$ for all $r>0$. If there exists $C>0$ such that

$$
\left\{\int_{0}^{\infty}\left[u(x) \int_{r}^{\infty} f(t) d t\right]^{q} d x\right\}^{\frac{1}{q}} \geq C\left\{\int_{0}^{\infty}[v(x) f(x)]^{p} d x\right\}^{\frac{1}{p}}
$$

holds for all admissible $f$, then

$$
\inf _{r>0} K(r)=\inf _{r>0}\left[\int_{0}^{r} u^{q}(x) d x\right]^{\frac{1}{q}}\left[\int_{0}^{r} v^{-p^{\prime}}(x) d x\right]^{\frac{1}{p^{\prime}}}=B>0 .
$$

Moreover if $0<q \leq p<1, K(r)$ not only satisfies (1.13) but also is non-decreasing, then (1.12) holds for all admissible $f$ with $B \geq C \geq p^{\frac{1}{q}}\left|p^{\prime}\right|^{\frac{1}{p^{\prime}}} B$.

The above theorems are all programmatic for the weighted mixed-norm Hardy inequalities. Although these theorems do not give the best constants, we intend to solve this for some weighted mixed-norm Hardy inequalities based on these theorems.

In [8], $\mathrm{Li}$ and Mao obtained the following conclusion by combining with the measure theory:

Theorem 1.4 Let $1<p \leq q<\infty, \mu$ and $v$ be two $\sigma$-finite Borel measures on $R$. Set

$$
B=\sup _{x \in R}(v(-\infty, x])^{\frac{1}{p^{\prime}}}(\mu[x, \infty))^{\frac{1}{q}},
$$

where $p^{\prime}=\frac{p}{p-1}$ is the conjugate number of $p$. If $A$ is the optimal constant such that for all $f: R \rightarrow R$,

$$
\left[\int_{R}\left|\int_{(-\infty, x)} f(y) v(d y)\right|^{q} \mu(d x)\right]^{\frac{1}{q}} \leq A\left[\int_{R}|f(x)|^{p} v(d x)\right]^{\frac{1}{p}}
$$

then

$$
B \leq A \leq k_{q, p} B
$$

where $k_{q, p}=\left(\frac{r}{B\left(\frac{1}{r}, \frac{q-1}{r}\right)}\right)^{\frac{1}{p}-\frac{1}{q}}, r=\frac{q}{p}-1$. 
There are other results on the mixed-norm Hardy inequalities, one can see [9-14].

Now, let $1<p<q<\infty$ and $\lambda \neq 1$. According to Theorem 1.1, there exists a constant $C(p, q, \lambda)>0$, such that

$$
\left\{\int_{0}^{\infty} x^{q(\lambda-1)-1} F^{q}(x) d x\right\}^{\frac{1}{q}} \leq C(p, q, \lambda)\left[\int_{0}^{\infty} x^{p \lambda-1} f^{p}(x) d x\right]^{\frac{1}{p}}
$$

holds for all admissible $f \geq 0$, where

$$
F(x)= \begin{cases}\int_{0}^{x} f(t) d t, & \lambda<1 \\ \int_{x}^{\infty} f(t) d t, & \lambda>1\end{cases}
$$

Base on the inequality (1.1), when $\lambda=\frac{1}{p}$, the best constant in the inequality (1.14) is

$$
C(p, q, \lambda)=\frac{1}{q-r-1}\left[\frac{r \Gamma\left(\frac{q}{r}\right)}{\Gamma\left(\frac{1}{r}\right) \Gamma\left(\frac{q-1}{r}\right)}\right]^{r},
$$

and the corresponding condition of equality is

$$
F(x)=\frac{x}{\left(\alpha x^{r}+\beta\right)^{\frac{1}{r}}} \quad(\alpha, \beta>0) .
$$

According to Theorem 1.1, $B$ is a lower bound, while $B p^{\frac{1}{q}}\left(p^{\prime}\right)^{\frac{1}{p^{\prime}}}$ is an upper bound of the best constant $C$. However, the best constant $C$ and the corresponding condition of equality are not determined, which is still an open problem $[15,16]$.

We will solve the above problem in this paper. Our main conclusions are as follows:

Theorem 1.5 Suppose that $1<p<q<\infty, \lambda \neq 1$ is a constant, $f(x)$ is non-negative and measurable in $(0, \infty)$, such that $0<\int_{0}^{\infty} x^{p \lambda-1} f^{p}(x) d x<\infty$. Let $r=\frac{q}{p}-1$ and

$$
y(x)= \begin{cases}\int_{0}^{x} f(t) d t, & \lambda<1 \\ \int_{x}^{\infty} f(t) d t, & \lambda>1\end{cases}
$$

then we have

(1) There exists the following inequality

$$
\begin{aligned}
& \int_{0}^{\infty} x^{q(\lambda-1)-1} y^{q}(x) d x \\
& \quad \leq \frac{1}{q-r-1}\left(\frac{p-1}{p|1-\lambda|}\right)^{q-r}\left[\frac{r \Gamma\left(\frac{q}{r}\right)}{\Gamma\left(\frac{1}{r}\right) \Gamma\left(\frac{q-1}{r}\right)}\right]^{r}\left[\int_{0}^{\infty} x^{p \lambda-1} f^{p}(x) d x\right]^{\frac{q}{p}} .
\end{aligned}
$$

(2) If and only if there exists two positive constants $c, d$, such that

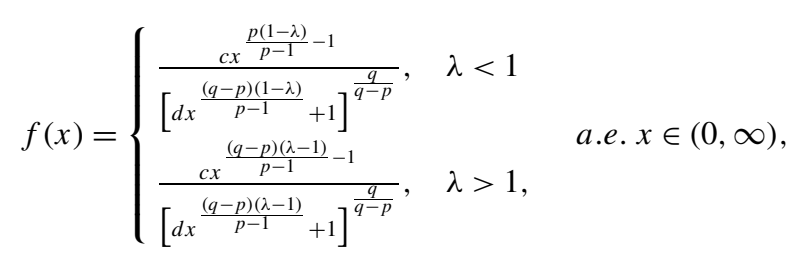

the equality holds. 
This paper is organized as follows. In Sect. 2, we introduce some necessary notations and preknowledge. In Sect. 3, we give the corresponding Euler equation which the optimal function satisfies, and conjecture the best constant and optimal function. In Sect. 4, we introduce an important auxiliary function $\phi$ and derive its some properties. In Sect. 5, an important auxiliary function $W$ is introduced and its some basic properties are given. In Sect. 6, we prove the inequality when $f$ is a continuous function. In Sect. 7, the equality condition of the inequality is determined in the continuous case. In Sect. 8, we prove the inequality when $f$ is a measurable function and determine the equality condition.

\section{Some Necessary Notations and Preknowledge}

From now on, we suppose that $1<p<q<\infty$ are two constants, and $r=\frac{q}{p}-1$.

For any constant $\lambda \neq 1$, we denote $E(\lambda)$ as the set of all non-negative and measurable function $f$ in $(0, \infty)$, which satisfies $0<\int_{0}^{\infty} x^{p \lambda-1} f^{p}(x) d x<\infty$. We further define a functional in $E(\lambda)$ as:

$$
J(f)= \begin{cases}\frac{\int_{0}^{\infty} x^{q(\lambda-1)-1}\left[\int_{0}^{x} f(t) d t\right]^{q} d x}{\left[\int_{0}^{\infty} x^{p \lambda-1} f^{p}(x) d x\right]^{\frac{q}{p}}}, & \lambda<1 \\ \frac{\int_{0}^{\infty} x^{q(\lambda-1)-1}\left[\int_{x}^{\infty} f(t) d t\right]^{q} d x}{\left[\int_{0}^{\infty} x^{p \lambda-1} f^{p}(x) d x\right]^{\frac{q}{p}}}, & \lambda>1,\end{cases}
$$

and

$$
C(p, q, \lambda)=\sup _{\lambda \in E(\lambda)} J(f)
$$

In fact, $C(p, q, \lambda)$ is the best constant in the inequality (1.14).

For any constant $\lambda \neq 1$, we denote

$$
k(p, q, \lambda)=\frac{1}{q-r-1}\left(\frac{p-1}{p|1-\lambda|}\right)^{q-r}\left[\frac{r \Gamma\left(\frac{q}{r}\right)}{\Gamma\left(\frac{1}{r}\right) \Gamma\left(\frac{q-1}{r}\right)}\right]^{r},
$$

then $k(p, q, \lambda)=k(p, q, 2-\lambda)$.

If $a>0, b>0, c>0$, we denote $I(a, b, c)=\int_{0}^{\infty} \frac{x^{a-1}}{\left(1+x^{b}\right)^{c}} d x$. It's easy to know that $I(a, b, c)$ converges if and only if $b c>a$. When $b c>a$, we obtain

$$
\begin{aligned}
I(a, b, c) & =\int_{0}^{\infty} \frac{x^{a-1}}{\left(1+x^{b}\right)^{c}} d x=\frac{1}{b} \int_{0}^{1} y^{c-\frac{a}{b}-1}(1-y)^{\frac{a}{b}-1} d y=\frac{1}{b} B\left(c-\frac{a}{b}, \frac{a}{b}\right) \\
& =\frac{\Gamma\left(c-\frac{a}{b}\right) \Gamma\left(\frac{a}{b}\right)}{b \Gamma(c)} .
\end{aligned}
$$

Finally, we denote $K=\{(x, y, z) \mid x>0, y>0, z>0\}$.

Now, let's introduce some necessary lemmas.

Lemma 2.1 ([17]) Suppose that $\lambda \neq 1$ is a constant. Let $f$ be a non-negative and measurable function in $(0, \infty)$, and $\int_{0}^{\infty} x^{p \lambda-1} f^{p}(x) d x<\infty$. Denote $y(x)=\left\{\begin{array}{l}\int_{0}^{x} f(t) d t, \quad \lambda<1 \\ \int_{x}^{\infty} f(t) d t, \lambda>1,\end{array}\right.$ 
then $y(x)$ is finite for every $x>0$. Moreover, the following limit formula holds

$$
\begin{cases}\lim _{x \rightarrow 0^{+}} \frac{y(x)}{x^{1-\lambda}}=0, & \lambda<1 \\ \lim _{x \rightarrow \infty} \frac{y(x)}{x^{1-\lambda}}=0, \quad \lambda>1\end{cases}
$$

The following lemma reveals the relation between the case $\lambda<1$ and the case $\lambda>1$ in the inequality (1.14). Based on this, the proof of Theorem 1.5 can be simplified.

Lemma 2.2 (1) For any $\lambda \neq 1$, we have the following equality

$$
C(p, q, \lambda)=C(p, q, 2-\lambda) .
$$

(2) For any $\lambda \neq 1$ and $f \in E(\lambda), J(f)=C(p, q, \lambda)$ if and only if $J(g)=C(p, q, 2-\lambda)$, where $g(x)=\frac{f\left(\frac{1}{x}\right)}{x^{2}}$.

Proof (1) Take an arbitrarily $\lambda<1$. For any $f \in E(\lambda)$, by setting $x=\frac{1}{u}$ and $t=\frac{1}{v}$, we have

$$
\int_{0}^{\infty} x^{q(\lambda-1)-1}\left[\int_{0}^{x} f(t) d t\right]^{q} d x=\int_{0}^{\infty} u^{q(2-\lambda-1)-1}\left[\int_{u}^{\infty} \frac{1}{v^{2}} f\left(\frac{1}{v}\right) d v\right]^{q} d u,
$$

and

$$
\int_{0}^{\infty} x^{p \lambda-1} f^{p}(x) d x=\int_{0}^{\infty} u^{p(2-\lambda)-1}\left[\frac{1}{u^{2}} f\left(\frac{1}{u}\right)\right]^{p} d u .
$$

On the other hand, for any $f \in E(2-\lambda)$, we also can obtain

$$
\int_{0}^{\infty} x^{q(2-\lambda-1)-1}\left[\int_{x}^{\infty} f(t) d t\right]^{q} d x=\int_{0}^{\infty} u^{q(\lambda-1)-1}\left[\int_{0}^{u} \frac{1}{v^{2}} f\left(\frac{1}{v}\right) d v\right]^{q} d u,
$$

and

$$
\int_{0}^{\infty} x^{p(2-\lambda)-1} f^{p}(x) d x=\int_{0}^{\infty} u^{p \lambda-1}\left[\frac{1}{u^{2}} f\left(\frac{1}{u}\right)\right]^{p} d u .
$$

For any $f \in E(\lambda)$, based on (2.3), (2.1) and (2.4), we can get

$$
\begin{aligned}
\int_{0}^{\infty} x^{q(\lambda-1)-1}\left[\int_{0}^{x} f(t) d t\right]^{q} d x & =\int_{0}^{\infty} u^{q(2-\lambda-1)-1}\left[\int_{u}^{\infty} \frac{1}{v^{2}} f\left(\frac{1}{v}\right) d v\right]^{q} d u \\
& \leq C(p, q, 2-\lambda)\left\{\int_{0}^{\infty} u^{p(2-\lambda)-1}\left[\frac{1}{u^{2}} f\left(\frac{1}{u}\right)\right]^{p} d u\right\}^{\frac{q}{p}} \\
& =C(p, q, 2-\lambda)\left[\int_{0}^{\infty} x^{p \lambda-1} f^{p}(x) d x\right]^{\frac{q}{p}}
\end{aligned}
$$

Therefore, $C(p, q, \lambda) \leq C(p, q, 2-\lambda)$. 
On the other hand, according to (2.5), (2.1) and (2.6), we have

$$
\begin{aligned}
\int_{0}^{\infty} x^{q(2-\lambda-1)-1}\left[\int_{x}^{\infty} f(t) d t\right]^{q} d x & =\int_{0}^{\infty} u^{q(\lambda-1)-1}\left[\int_{0}^{u} \frac{1}{v^{2}} f\left(\frac{1}{v}\right) d v\right]^{q} d u \\
& \leq C(p, q, \lambda)\left\{\int_{0}^{\infty} u^{p \lambda-1}\left[\frac{1}{u^{2}} f\left(\frac{1}{u}\right)\right]^{p} d u\right\}^{\frac{q}{p}} \\
& =C(p, q, \lambda)\left\{\int_{0}^{\infty} x^{p(2-\lambda)-1} f^{p}(x) d x\right\}^{\frac{q}{p}} .
\end{aligned}
$$

Therefore, $C(p, q, 2-\lambda) \leq C(p, q, \lambda)$.

In summary, for any $\lambda<1, C(p, q, \lambda)=C(p, q, 2-\lambda)$. For any $\lambda>1,2-\lambda<1$. By replacing $\lambda$ with $2-\lambda$ in the last equality, we can get $C(p, q, 2-\lambda)=C(p, q, \lambda), \lambda>1$. Therefore, for any $\lambda \neq 1$, we have $C(p, q, \lambda)=C(p, q, 2-\lambda)$.

(2) If $\lambda<1$, based on (2.3), (2.4) and (2.2), $J(f)=C(p, q, \lambda)$ if and only if

$$
\begin{aligned}
& \int_{0}^{\infty} x^{q(2-\lambda-1)-1}\left[\int_{x}^{\infty} \frac{1}{t^{2}} f\left(\frac{1}{t}\right) d t\right]^{q} d x \\
& =C(p, q, \lambda)\left\{\int_{0}^{\infty} x^{p(2-\lambda)-1}\left[\frac{1}{x^{2}} f\left(\frac{1}{x}\right)\right]^{p} d x\right\}^{\frac{q}{p}} \\
& =C(p, q, 2-\lambda)\left\{\int_{0}^{\infty} x^{p(2-\lambda)-1}\left[\frac{1}{x^{2}} f\left(\frac{1}{x}\right)\right]^{p} d x\right\}^{\frac{q}{p}},
\end{aligned}
$$

namely $J(g)=C(p, q, 2-\lambda)$.

If $\lambda>1$, based on (2.5), (2.6) and (2.2), $J(f)=C(p, q, \lambda)$ if and only if

$$
\begin{aligned}
& \int_{0}^{\infty} x^{q(2-\lambda-1)-1}\left[\int_{0}^{x} \frac{1}{t^{2}} f\left(\frac{1}{t}\right) d t\right]^{q} d x \\
& =C(p, q, \lambda)\left\{\int_{0}^{\infty} x^{p(2-\lambda)-1}\left[\frac{1}{x^{2}} f\left(\frac{1}{x}\right)\right]^{p} d x\right\}^{\frac{q}{p}} \\
& =C(p, q, 2-\lambda)\left\{\int_{0}^{\infty} x^{p(2-\lambda)-1}\left[\frac{1}{x^{2}} f\left(\frac{1}{x}\right)\right]^{p} d x\right\}^{\frac{q}{p}},
\end{aligned}
$$

namely $J(g)=C(p, q, 2-\lambda)$.

\section{The Corresponding Euler Equation}

With the help of Lemma 2.2, we only need to study the case of $\lambda<1$. Throughout Sects. 3-8, $\lambda<1$ is always assumed. Now we introduce and prove a key lemma.

Lemma 3.1 Suppose that $\lambda<1, f \in E(\lambda) \cap C(0, \infty)$ and satisfies $J(f)=C(p, q, \lambda)$. Let

$$
y(x)=\int_{0}^{x} f(t) d t, \quad x \in[0, \infty), \quad A=-\frac{\int_{0}^{\infty} x^{p \lambda-1} y^{\prime p}(x) d x}{\int_{0}^{\infty} x^{q(\lambda-1)-1} y^{q}(x) d x},
$$

then 
(1) $y^{\prime}(x)>0, x>0$

(2) $\left[x^{p \lambda-1} y^{\prime p-1}(x)\right]^{\prime}=A x^{q(\lambda-1)-1} y^{q-1}(x), x>0$.

Proof For any $\theta>0$ and $x_{0}>0$, let $g(x)=\left\{\begin{array}{l}\theta f(x), x \in\left(0, x_{0}\right] \\ f(x), x>x_{0},\end{array}\right.$ then $g \in E(\lambda)$. Set $z(x)=$ $\int_{0}^{x} g(t) d t$, then

$$
z(x)= \begin{cases}\theta y(x) & x \in\left[0, x_{0}\right] \\ y(x)+(\theta-1) y\left(x_{0}\right), & x>x_{0}\end{cases}
$$

Set

$$
Q(\theta)=\frac{\int_{0}^{\infty} x^{q(\lambda-1)-1} z^{q}(x) d x}{\left[\int_{0}^{\infty} x^{p \lambda-1} z^{\prime p}(x) d x\right]^{\frac{q}{p}}}, \quad \theta>0,
$$

then $Q(\theta) \leq C(p, q, \lambda)$ and $Q(1)=C(p, q, \lambda)$. Therefore, $Q(\theta)$ achieves the maximum value at $\theta=1$.

By direct computation, we can get

$$
Q^{\prime}(\theta)=q \frac{\left\{\theta^{q-1} \int_{0}^{x_{0}} x^{q(\lambda-1)-1} y^{q}(x) d x+y\left(x_{0}\right) \int_{x_{0}}^{\infty} x^{q(\lambda-1)-1}\left[y(x)+(\theta-1) y\left(x_{0}\right)\right]^{q-1} d x\right\}}{\left[\int_{0}^{\infty} x^{p \lambda-1} z^{\prime p}(x) d x\right]^{\frac{q}{p}+1}} .
$$

Base on Fermat Lemma, we have $Q^{\prime}(1)=0$, that is

$$
-A\left\{\int_{0}^{x_{0}} x^{q(\lambda-1)-1} y^{q}(x) d x+y\left(x_{0}\right) \int_{x_{0}}^{\infty} x^{q(\lambda-1)-1} y^{q-1}(x) d x\right\}=\int_{0}^{x_{0}} x^{p \lambda-1} y^{\prime p}(x) d x .
$$

Take derivatives on both side of (3.1) with respect to $x_{0}$, we can get

$$
-A y^{\prime}\left(x_{0}\right) \int_{x_{0}}^{\infty} x^{q(\lambda-1)-1} y^{q-1}(x) d x=x_{0}^{p \lambda-1} y^{\prime p}\left(x_{0}\right) .
$$

Therefore, if $y^{\prime}\left(x_{0}\right)>0$, then

$$
-A \int_{x_{0}}^{\infty} x^{q(\lambda-1)-1} y^{q-1}(x) d x=x_{0}^{p \lambda-1} y^{\prime p-1}\left(x_{0}\right)
$$

Now, we point out that there is no $x_{0}>0$ to satisfy the following property $(p)$ :

(i) $y^{\prime}\left(x_{0}\right)=0$;

(ii) There exist a sequence $\left\{x_{n}\right\} \subset(0, \infty)$, such that $\lim _{n \rightarrow \infty} x_{n}=x_{0}$ and $y^{\prime}\left(x_{n}\right)>0, n=$ $1,2, \ldots$

In fact, let's assume that $x_{0}>0$ satisfies the property $(p)$, then $y^{\prime}\left(x_{0}\right)=0$. Let the positive sequence $\left\{x_{n}\right\}$ satisfy $\lim _{n \rightarrow \infty} x_{n}=x_{0}$ and $y^{\prime}\left(x_{n}\right)>0, n=1,2, \ldots$, then

$$
-A \int_{x_{n}}^{\infty} x^{q(\lambda-1)-1} y^{q-1}(x) d x=x_{n}^{p \lambda-1} y^{\prime p-1}\left(x_{n}\right) .
$$


Let $n \rightarrow \infty$ on both side of the above equality, we can get

$$
-A \int_{x_{0}}^{\infty} x^{q(\lambda-1)-1} y^{q-1}(x) d x=x_{0}^{p \lambda-1} y^{\prime p-1}\left(x_{0}\right)=0 .
$$

Since $A<0, x^{q(\lambda-1)-1} y^{q-1}(x)$ is non-negative and continuous on $\left[x_{0}, \infty\right)$, then

$$
x^{q(\lambda-1)-1} y^{q-1}(x)=0, \quad x \in\left[x_{0}, \infty\right),
$$

which means $y(x)=0, x \in\left[x_{0}, \infty\right)$. But $y(x)$ is non-decreasing on $[0, \infty)$ and $y(0)=0$, so $y(x)=0, x \in[0, \infty)$. Therefore, $f(x)=y^{\prime}(x)=0, x \in(0, \infty)$. Then $\int_{0}^{\infty} x^{p \lambda-1} f^{p}(x) d x=0$, which is a contradiction. Therefore, there is no $x_{0}>0$ to satisfy the property $(p)$.

Let's assume that there is a $x_{0}>0$, such that $y^{\prime}\left(x_{0}\right)=0$. As we know, $x_{0}$ can't satisfy the property $(p)$, so there exists a $\delta>0$, such that $y^{\prime}(x)=0, x \in\left(x_{0}-\delta, x_{0}+\delta\right)$. Let $(a, b)$ be the largest open interval which contains $x_{0}$ and satisfies $y^{\prime}(x)=0$ in it, then $a>0$ or $b<\infty$. If $a>0$, then $y^{\prime}(a)=0$ and there exists a sequence $\left\{x_{n}\right\} \subset(0, a)$, such that $\lim _{n \rightarrow \infty} x_{n}=a$ and $y^{\prime}\left(x_{n}\right)>0$. Therefore, $a$ satisfies the property $(p)$, which is a contradiction. If $b<\infty$, then $y^{\prime}(b)=0$ and there exists a sequence $\left\{x_{n}\right\} \subset(b, \infty)$, such that $\lim _{n \rightarrow \infty} x_{n}=b$ and $y^{\prime}\left(x_{n}\right)>0$. Therefore, $b$ satisfies the property $(p)$, which is also a contradiction. Therefore, $y^{\prime}(x)>0$, $x \in(0, \infty)$. Thus, we have proved (1).

As $y^{\prime}(x)>0, x \in(0, \infty)$, based on (3.2), we have

$$
-A \int_{x}^{\infty} t^{q(\lambda-1)-1} y^{q-1}(t) d t=x^{p \lambda-1} y^{p-1}(x) .
$$

Take derivatives on both side of the above equality, we can get

$$
\left[x^{p \lambda-1} y^{\prime p-1}(x)\right]^{\prime}=A x^{q(\lambda-1)-1} y^{q-1}(x) .
$$

Thus, we have proved (2) and completed the proof of the lemma.

Now we give a solution of the following Cauchy problem:

$$
\left\{\begin{array}{l}
{\left[x^{p \lambda-1} y^{\prime p-1}(x)\right]^{\prime}=A x^{q(\lambda-1)-1} y^{q-1}(x), \quad x>0} \\
y(0)=0,
\end{array}\right.
$$

where $A$ is a negative constant. We further demand that $y^{\prime}(x)>0, x \in(0, \infty)$ and $\int_{0}^{\infty} x^{q(\lambda-1)} y^{q}(x) d x<\infty$.

Let $y(x)=\gamma \frac{x^{\alpha \beta}}{\left(1+x^{\alpha}\right)^{\beta}}$ be a solution of (3.3), where $\alpha, \beta, \gamma$ are pending and positive numbers. By direct calculation, we can obtain

$$
\begin{aligned}
& \alpha=\frac{(q-p)(1-\lambda)}{p-1}, \quad \beta=\frac{p}{q-p}, \quad \gamma=\left\{-\frac{A}{q(1-\lambda)}\left[\frac{p-1}{p(1-\lambda)}\right]^{p-1}\right\}^{\frac{1}{p-q}}, \\
& y(x)=\left\{-\frac{A}{q(1-\lambda)}\left[\frac{p-1}{p(1-\lambda)}\right]^{p-1}\right\}^{\frac{1}{p-q}} \frac{x^{\frac{p(1-\lambda)}{p-1}}}{\left(1+x^{\frac{(q-p)(1-\lambda)}{p-1}}\right)^{\frac{p}{q-p}}} .
\end{aligned}
$$




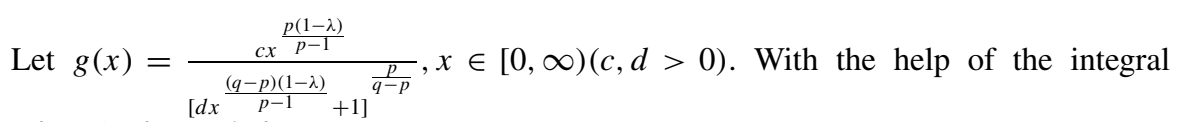
$I(a, b, c)(a, b, c>0, b c>a)$, we can get

$$
\frac{\int_{0}^{\infty} x^{q(\lambda-1)-1} g^{q}(x) d x}{\left[\int_{0}^{\infty} x^{p \lambda-1} g^{\prime} p(x) d x\right]^{\frac{q}{p}}}=\frac{1}{q-r-1}\left[\frac{p-1}{p(1-\lambda)}\right]^{q-r}\left[\frac{r \Gamma\left(\frac{q}{r}\right)}{\Gamma\left(\frac{q-1}{r}\right) \Gamma\left(\frac{1}{r}\right)}\right]^{r}=k(p, q, \lambda) .
$$

Thus, we can conjecture that $k(p, q, \lambda)$ is the best constant, while $g^{\prime}(x)$ is an optimal function.

\section{Auxiliary Function $\phi$}

Next, we start to prove Theorem 1.5. In this section, we introduce an important auxiliary function $\phi$.

Let $\phi$ be the function defined by the following equation

$$
\phi(u)=\frac{1}{(1-u)^{\frac{1}{r}} u^{\frac{p-1}{r}}} \int_{0}^{\left(\frac{u}{1-u}\right)^{\frac{p-1}{(q-p)(1-\lambda)}}} \frac{\zeta^{\frac{p(q-1)(1-\lambda)}{p-1}-1}}{\left[\zeta^{\frac{(q-p)(1-\lambda)}{p-1}}+1\right]^{\frac{p q}{q-p}}} d \zeta, \quad u \in(0,1) .
$$

Let's discuss some related problems about $\phi$.

(a) From (4.1), the following facts can be known.

(i) There is a continuous derivative of $\phi(u)$ in $(0,1)$ :

$$
\phi^{\prime}(u)=\frac{p u-p+1}{r u(1-u)} \phi(u)+\frac{p-1}{(q-p)(1-\lambda)} \frac{u^{p-1}}{1-u}, \quad u \in(0,1) .
$$

(ii) When $u \rightarrow 0^{+}$, there is an equivalent infinitesimal of $\phi(u)$ :

$$
\phi(u) \sim \frac{p-1}{p(q-1)(1-\lambda)} u^{p}, \quad u \rightarrow 0^{+} .
$$

So we can supplementary agree that $\phi(0)=0$.

(iii) $\lim _{u \rightarrow 1^{-}} \phi(u)=\infty$.

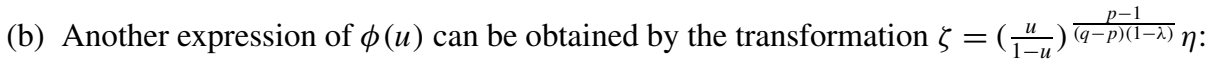

$$
\phi(u)=u^{p} \int_{0}^{1} \frac{\eta^{\frac{p(q-1)(1-\lambda)}{p-1}-1}}{\left[1-u+u \eta^{\frac{(q-p)(1-\lambda)}{p-1}}\right]^{\frac{p q}{q-p}}} d \eta, \quad u \in(0,1)
$$

$\phi^{\prime}(u)>0, u \in(0,1)$ can be obtained from (4.4) easily.

(c) $\phi(u)$ is strictly increasing and continuous on $[0,1)$ and $\phi(0)=0, \lim _{u \rightarrow 1^{-}} \phi(u)=\infty$. Thus for any $(x, y, z) \in K$, the equation $\phi(u)=\left[\frac{p-1}{p(1-\lambda)}\right]^{p} x^{p(1-\lambda)} y^{-p} z$ has a unique solution in $(0,1)$, which can be recorded as

$$
u=u(x, y, z)=\phi^{-1}\left\{\left[\frac{p-1}{p(1-\lambda)}\right]^{p} x^{p(1-\lambda)} y^{-p} z\right\} .
$$


Based on the implicit function theorem, $u(x, y, z)$ is continuously differentiable in $K$ and satisfies:

$$
\begin{aligned}
\phi^{\prime}(u) \frac{\partial u}{\partial x} & =p(1-\lambda)\left[\frac{p-1}{p(1-\lambda)}\right]^{p} x^{p(1-\lambda)-1} y^{-p} z \\
\phi^{\prime}(u) \frac{\partial u}{\partial y} & =-p\left[\frac{p-1}{p(1-\lambda)}\right]^{p} x^{p(1-\lambda)} y^{-p-1} z \\
\phi^{\prime}(u) \frac{\partial u}{\partial z} & =\left[\frac{p-1}{p(1-\lambda)}\right]^{p} x^{p(1-\lambda)} y^{-p}
\end{aligned}
$$

(d) Set

$$
\left\{\begin{array}{l}
y(x)=\frac{c x^{\frac{p(1-\lambda)}{p-1}}}{\left[d x^{\frac{(q-p)(1-\lambda)}{p-1}}+1\right]^{\frac{p}{q-p}}} \\
z(x)=\int_{x}^{\infty} t^{p \lambda-1}\left[y^{\prime}(t)\right]^{p} d t=\left[\frac{p(1-\lambda)}{p-1}\right]^{p} \int_{x}^{\infty} \frac{c^{p} t^{\frac{p(1-\lambda)}{p-1}-1}}{\left[d t^{\frac{(q-p)(1-\lambda)}{p-1}}+1\right]^{\frac{p q}{q-p}}} d t,
\end{array} \quad x \in[0, \infty)\right.
$$

where $c, d>0$. We can get

$$
\left[\frac{p-1}{p(1-\lambda)}\right]^{p} x^{p(1-\lambda)} y^{-p}(x) z(x)=\phi\left(\frac{1}{d x^{\frac{(q-p)(1-\lambda)}{p-1}}+1}\right), \quad x \in(0, \infty),
$$

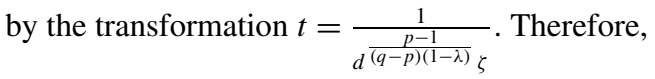

$$
u(x, y(x), z(x))=\frac{1}{d x^{\frac{(q-p)(1-\lambda)}{p-1}}+1}, \quad x \in(0, \infty) .
$$

Furthermore,

$$
\begin{aligned}
& \left.y^{\prime}(x)=\frac{p(1-\lambda)}{p-1} \frac{c x^{\frac{p(1-\lambda)}{p-1}}}{\left(d x \frac{(q-p)(1-\lambda)}{p-1}\right.}+1\right)^{\frac{p}{q-p}} \frac{1}{x\left(d x^{\frac{(q-p)(1-\lambda)}{p-1}}+1\right)}=\frac{p(1-\lambda)}{p-1} \frac{u y}{x}, \quad x>0, \\
& z^{\prime}(x)=-x^{p \lambda-1}\left[y^{\prime}(x)\right]^{p}=-x^{p \lambda-1}\left[\frac{p(1-\lambda)}{p-1} \frac{u y}{x}\right]^{p}, \quad x>0 .
\end{aligned}
$$

Therefore, $y(x), z(x)$ satisfy the following ordinary differential system:

$$
\left\{\begin{array}{l}
y^{\prime}(x)=\frac{p(1-\lambda)}{p-1} \frac{u y}{x} \\
z^{\prime}(x)=-x^{p \lambda-1}\left[\frac{p(1-\lambda)}{p-1} \frac{u y}{x}\right]^{p}, \quad x>0 .
\end{array}\right.
$$

(e) We are going to derive some limits relating to $u$.

(i) For any $x_{1}>0$,

$$
\lim _{(x, y, z) \rightarrow\left(x_{1}, 0,1\right)} \phi(u)=\lim _{(x, y, z) \rightarrow\left(x_{1}, 0,1\right)}\left[\frac{p-1}{p(1-\lambda)}\right]^{p} x^{p(1-\lambda)} y^{-p} z=\infty,
$$


so $\lim _{(x, y, z) \rightarrow\left(x_{1}, 0,1\right)} u=1$.

$$
\begin{aligned}
\phi(u) & =\frac{1}{(1-u)^{\frac{1}{r}} u^{\frac{p-1}{r}}} \int_{0}^{\left(\frac{u}{1-u}\right)^{\frac{p-1}{(q-p)(1-\lambda)}}} \frac{\zeta^{\frac{p(q-1)(1-\lambda)}{p-1}-1}}{\left[\zeta^{\frac{(q-p)(1-\lambda)}{p-1}}+1\right]^{\frac{p q}{q-p}} d \zeta} \\
& =\left[\frac{p-1}{p(1-\lambda)}\right]^{p} x^{p(1-\lambda)} y^{-p} z,
\end{aligned}
$$

that is

$$
\int_{0}^{\left(\frac{u}{1-u}\right)^{\frac{p-1}{(q-p)(1-\lambda)}}} \frac{\zeta^{\frac{p(q-1)(1-\lambda)}{p-1}-1}}{\left[\zeta^{\frac{(q-p)(1-\lambda)}{p-1}}+1\right]^{\frac{p q}{q-p}}} d \zeta=\left[\frac{p-1}{p(1-\lambda)}\right]^{p} x^{p(1-\lambda)} y^{-p} z(1-u)^{\frac{1}{r}} u^{\frac{p-1}{r}}
$$

Therefore,

$$
\begin{aligned}
& \lim _{(x, y, z) \rightarrow\left(x_{1}, 0,1\right)}\left[\frac{p-1}{p(1-\lambda)}\right]^{p} x^{p(1-\lambda)} y^{-p} z(1-u)^{\frac{1}{r}} u^{\frac{p-1}{r}} \\
& =\lim _{(x, y, z) \rightarrow\left(x_{1}, 0,1\right)} \int_{0}^{\left(\frac{u}{1-u}\right)^{\frac{p-1}{(q-p)(1-\lambda)}}} \frac{\zeta^{\frac{p(q-1)(1-\lambda)}{p-1}-1}}{\left[\zeta^{\frac{(q-p)(1-\lambda)}{p-1}}+1\right]^{\frac{p q}{q-p}} d \zeta} \\
& =\int_{0}^{\infty} \frac{\zeta^{\frac{p(q-1)(1-\lambda)}{p-1}-1}}{\left[\zeta^{\frac{(q-p)(1-\lambda)}{p-1}}+1\right]^{\frac{p q}{q-p}}} d \zeta=I\left(\frac{p(q-1)(1-\lambda)}{p-1}, \frac{(q-p)(1-\lambda)}{p-1}, \frac{p q}{q-p}\right) \\
& =\frac{p-1}{p(1-\lambda)} \frac{\Gamma\left(\frac{1}{r}\right) \Gamma\left(\frac{q-1}{r}\right)}{r \Gamma\left(\frac{q}{r}\right)} .
\end{aligned}
$$

Thus,

$$
\lim _{(x, y, z) \rightarrow\left(x_{1}, 0,1\right)}\left[\frac{p-1}{p(1-\lambda)}\right]^{p} x^{p(1-\lambda)} y^{-p}(1-u)^{\frac{1}{r}} u^{\frac{p-1}{r}}=\frac{p-1}{p(1-\lambda)} \frac{\Gamma\left(\frac{1}{r}\right) \Gamma\left(\frac{q-1}{r}\right)}{r \Gamma\left(\frac{q}{r}\right)} .
$$

(ii) Suppose that $f$ is non-negative and measurable in $(0, \infty)$ and satisfies $\int_{0}^{\infty} x^{p \lambda-1} f^{p}(x) d x=1$ and $\int_{0}^{x} f(t) d t>0, x>0$. Let $\left\{\begin{array}{l}y(x)=\int_{0}^{x} f(t) d t \\ z(x)=\int_{x}^{\infty} t^{p \lambda-1} f^{p}(t) d t,\end{array}\right.$ $x>0$, then $y(x)>0$ and $\lim _{x \rightarrow 0^{+}} z(x)=1$. Based on Lemma 2.1, we can get $\lim _{x \rightarrow 0^{+}} \frac{y(x)}{x^{1-\lambda}}=0$, thus $\lim _{x \rightarrow 0^{+}} x^{p(1-\lambda)} y^{-p}(x) z(x)=\infty$. Then we also can get

$$
\lim _{x \rightarrow 0^{+}}\left[\frac{p-1}{p(1-\lambda)}\right]^{p} x^{p(1-\lambda)} y^{-p}(x)(1-u)^{\frac{1}{r}} u^{\frac{p-1}{r}}=\frac{p-1}{p(1-\lambda)} \frac{\Gamma\left(\frac{1}{r}\right) \Gamma\left(\frac{q-1}{r}\right)}{r \Gamma\left(\frac{q}{r}\right)} .
$$

(iii) For any $x_{2}, y_{2}>0$,

$$
\lim _{(x, y, z) \rightarrow\left(x_{2}, y_{2}, 0\right)} \phi(u)=\lim _{(x, y, z) \rightarrow\left(x_{2}, y_{2}, 0\right)}\left[\frac{p-1}{p(1-\lambda)}\right]^{p} x^{p(1-\lambda)} y^{-p} z=0,
$$


so $\lim _{(x, y, z) \rightarrow\left(x_{2}, y_{2}, 0\right)} u=0$.

$$
\phi(u)=\left[\frac{p-1}{p(1-\lambda)}\right]^{p} x^{p(1-\lambda)} y^{-p} z,
$$

therefore,

$$
\begin{aligned}
\lim _{(x, y, z) \rightarrow\left(x_{2}, y_{2}, 0\right)} \frac{\phi(u)}{z} & =\lim _{(x, y, z) \rightarrow\left(x_{2}, y_{2}, 0\right)}\left[\frac{p-1}{p(1-\lambda)}\right]^{p} x^{p(1-\lambda)} y^{-p} \\
& =\left[\frac{p-1}{p(1-\lambda)}\right]^{p} \frac{x_{2}^{p(1-\lambda)}}{y_{2}^{p}} .
\end{aligned}
$$

From (4.3), $\phi(u) \sim \frac{p-1}{p(q-1)(1-\lambda)} u^{p}, u \rightarrow 0^{+}$, then

$$
\lim _{(x, y, z) \rightarrow\left(x_{2}, y_{2}, 0\right)} \frac{u^{p}}{z}=(q-1)\left[\frac{p-1}{p(1-\lambda)}\right]^{p-1} \frac{x_{2}^{p(1-\lambda)}}{y_{2}^{p}} .
$$

\section{Auxiliary Function $W$}

In this section, we construct an auxiliary function $W$ based on $\phi$, and determine some limits and partial derivatives of $W$.

The following equation

$$
\begin{aligned}
& W=-\left\{\frac{1}{q(1-\lambda)} \frac{1}{1-u} \frac{y^{q}}{x^{q(1-\lambda)}}+\frac{1}{q-r-1}\left[\frac{p-1}{p(1-\lambda)}\right]^{p} \frac{1}{(1-u) u^{p-1}} \frac{y^{q-p} z}{x^{(q-p)(1-\lambda)}}\right\}, \\
& (x, y, z) \in K,
\end{aligned}
$$

determines a function $W(x, y, z)$ when $u$ in this expression is replaced by the function $u(x, y, z)=\phi^{-1}\left(\left[\frac{p-1}{p(1-\lambda)}\right]^{p} x^{p(1-\lambda)} y^{-p} z\right)$ described in the preceding section. It's easy to see that $W(x, y, z)<0, \forall(x, y, z) \in K$.

From (4.10), for any $x_{1}>0$, we have

$$
\lim _{(x, y, z) \rightarrow\left(x_{1}, 0,1\right)} W(x, y, z)=-k(p, q, \lambda) .
$$

Suppose that $f$ is non-negative and measurable in $(0, \infty)$, and satisfies $\int_{0}^{\infty} x^{p \lambda-1} f^{p}(x) d x=1$ and $\int_{0}^{x} f(t) d t>0, x>0$. Set $\left\{\begin{array}{l}y(x)=\int_{0}^{x} f(t) d t \\ z(x)=\int_{x}^{\infty} t^{p \lambda-1} f^{p}(t) d t,\end{array} \quad x \in\right.$ $[0, \infty)$. From (4.11), we can get

$$
\lim _{x \rightarrow 0^{+}} W(x, y(x), z(x))=-k(p, q, \lambda) .
$$

For any $x_{2}, y_{2}>0$, from (4.12), we can get

$$
\lim _{(x, y, z) \rightarrow\left(x_{2}, y_{2}, 0\right)} W(x, y, z)=-\frac{1}{q(1-\lambda)} \frac{y_{2}^{q}}{x_{2}^{q(1-\lambda)}} .
$$


If we treat $W$ as a function of the independent variables $x, y, z, u$, combining with (4.2), we can get the partial derivative of $W$ with respect to $u$ :

$$
\frac{\partial W}{\partial u}=-\frac{r}{q-r-1} \frac{1}{(1-u) u^{p-1}} \frac{y^{q}}{x^{q(1-\lambda)}} \phi^{\prime}(u) .
$$

Then, combining with the chain rule and (4.5)-(4.7), we can get the partial derivatives of $W$ with respect to $x, y, z$ :

$$
\begin{aligned}
W_{x} & =\frac{1}{1-u} \frac{y^{q}}{x^{q(1-\lambda)+1}}, \\
W_{y} & =-\frac{1}{1-\lambda} \frac{1}{1-u} \frac{y^{q-1}}{x^{q(1-\lambda)}}, \\
W_{z} & =-\frac{1}{p-1}\left[\frac{p-1}{p(1-\lambda)}\right]^{p} \frac{1}{(1-u) u^{p-1}} \frac{y^{q-p}}{x^{(q-p)(1-\lambda)}} .
\end{aligned}
$$

\section{Proof of the Inequality Under Continuous Condition}

Suppose that $f$ is non-negative and continuous in $(0, \infty)$, and satisfies $\int_{0}^{\infty} x^{p \lambda-1} f^{p}(x) d x=1$. We define two functions $y(x)$ and $z(x)$ as follows:

$$
\left\{\begin{array}{l}
y(x)=\int_{0}^{x} f(t) d t \\
z(x)=\int_{x}^{\infty} t^{p \lambda-1} f^{p}(t) d t,
\end{array} \quad x \in[0, \infty) .\right.
$$

We need to prove $\int_{0}^{\infty} x^{q(\lambda-1)-1} y^{q}(x) d x \leq k(p, q, \lambda)$.

Denote

$$
x_{1}=\sup \{x \in[0, \infty) \mid y(x)=0\}, \quad x_{2}=\inf \{x \in(0, \infty] \mid z(x)=0\},
$$

then $0 \leq x_{1}<x_{2} \leq \infty$. When $0 \leq x \leq x_{1}, y(x)=0, z(x)=1$; when $x_{1}<x<x_{2}$, $y(x)>0, z(x)>0$; when $x \geq x_{2}, y(x)$ is a positive constant, $z(x)=0$.

Set

$$
W(x)=W(x, y(x), z(x)), \quad x_{1}<x<x_{2} .
$$

According to the chain rule and (5.4)-(5.6), we have

$$
W^{\prime}(x)=\frac{y^{q}}{x^{q(1-\lambda)+1}}+\Psi(x),
$$

where

$$
\begin{aligned}
\Psi(x)= & \frac{p}{p-1} \frac{1}{(1-u) u^{p-1}} \frac{y^{q-p}}{x^{q(1-\lambda)+1}} \\
& \times\left\{\frac{p-1}{p}(u y)^{p}-(u y)^{p-1}\left[\frac{p-1}{p(1-\lambda)} x f\right]+\frac{1}{p}\left[\frac{p-1}{p(1-\lambda)} x f\right]^{p}\right\} .
\end{aligned}
$$


$W^{\prime}(x)$ is continuous in $\left(x_{1}, x_{2}\right)$. From Young inequality, $\Psi(x) \geq 0$ and $\Psi(x)=0$ if and only if $u y=\frac{p-1}{p(1-\lambda)} x f$, namely $y^{\prime}=\frac{p(1-\lambda)}{p-1} \frac{u y}{x}$. Thus, $W^{\prime}(x) \geq \frac{y^{q}}{x^{q(1-\lambda)+1}}$ and if and only if $y^{\prime}=\frac{p(1-\lambda)}{p-1} \frac{u y}{x}$, the equality holds.

If $x_{1}>0, \lim _{x \rightarrow x_{1}^{+}} W(x)=-k(p, q, \lambda)$ can be obtained due to (5.1). If $x_{1}=0, \lim _{x \rightarrow x_{1}^{+}} W(x)=$ $-k(p, q, \lambda)$ can be obtained due to (5.2). Thus, we always have $\lim _{x \rightarrow x_{1}^{+}} W(x)=-k(p, q, \lambda)$, from which we can supplementary agree that $W\left(x_{1}\right)=-k(p, q, \lambda)$.

If $x_{2}<\infty$, we denote $y\left(x_{2}\right)$ as $y_{2}$, then $y_{2}>0$. From (5.3), we have $\lim _{x \rightarrow x_{2}^{-}} W(x)=$ $-\frac{1}{q(1-\lambda)} \frac{y_{2}^{q}}{x_{2}^{q(1-\lambda)}}$, from which we can supplementary agree that $W\left(x_{2}\right)=-\frac{1}{q(1-\lambda)} \frac{y_{2}^{q}}{x_{2}^{q(1-\lambda)}}$. Therefore, we have

$$
\begin{aligned}
\int_{0}^{\infty} x^{q(\lambda-1)-1} y^{q}(x) d x & =\int_{x_{1}}^{x_{2}} x^{q(\lambda-1)-1} y^{q}(x) d x+\int_{x_{2}}^{\infty} x^{q(\lambda-1)-1} y^{q}(x) d x \\
& \leq \int_{x_{1}}^{x_{2}} W^{\prime}(x) d x+\frac{1}{q(1-\lambda)} \frac{y_{2}^{q}}{x_{2}^{q(1-\lambda)}} \\
& =W\left(x_{2}\right)-W\left(x_{1}\right)+\frac{1}{q(1-\lambda)} \frac{y_{2}^{q}}{x_{2}^{q(1-\lambda)}} \\
& =-\frac{1}{q(1-\lambda)} \frac{y_{2}^{q}}{x_{2}^{q(1-\lambda)}}+k(p, q, \lambda)+\frac{1}{q(1-\lambda)} \frac{y_{2}^{q}}{x_{2}^{q(1-\lambda)}}=k(p, q, \lambda) .
\end{aligned}
$$

If and only if $x^{q(\lambda-1)-1} y^{q}(x)=W^{\prime}(x)$, namely $y^{\prime}=\frac{p(1-\lambda)}{p-1} \frac{u y}{x}, x \in\left(x_{1}, x_{2}\right)$, the equality holds.

Now, we assume that $x_{2}=\infty$, then $W^{\prime}(x)>0, x \in\left(x_{1}, \infty\right)$. For any $A>x_{1}$, we have

$$
\int_{x_{1}}^{A} W^{\prime}(x) d x=W(A)-W\left(x_{1}\right)<-W\left(x_{1}\right)=k(p, q, \lambda) .
$$

Therefore, $\int_{x_{1}}^{\infty} W^{\prime}(x) d x$ converges and $\int_{x_{1}}^{\infty} W^{\prime}(x) d x \leq k(p, q, \lambda)$, thus

$$
\int_{0}^{\infty} x^{q(\lambda-1)-1} y^{q}(x) d x=\int_{x_{1}}^{\infty} x^{q(\lambda-1)-1} y^{q}(x) d x \leq \int_{x_{1}}^{\infty} W^{\prime}(x) d x \leq k(p, q, \lambda) .
$$

If the equality holds, then $x^{q(\lambda-1)-1} y^{q}(x)=W^{\prime}(x)$, namely $y^{\prime}=\frac{p(1-\lambda)}{p-1} \frac{u y}{x}, x \in\left(x_{1}, \infty\right)$.

In summary, we always have $\int_{0}^{\infty} x^{q(\lambda-1)-1} y^{q}(x) d x \leq k(p, q, \lambda)$. If the equality holds, $y^{\prime}=\frac{p(1-\lambda)}{p-1} \frac{u y}{x}, x \in\left(x_{1}, x_{2}\right)$.

Base on the homogeneity, for any $f \in E(\lambda) \cap C(0, \infty)$, we have $J(f) \leq k(p, q, \lambda)$. If the equality holds, the function $y(x)$ must satisfy $y^{\prime}=\frac{p(1-\lambda)}{p-1} \frac{u y}{x}, x \in\left(x_{1}, x_{2}\right)$.

\section{Proof for the Equality Condition in Continuous Case}

Suppose that $f$ is non-negative and continuous in $(0, \infty)$ and satisfies

$$
J(f)=\frac{\int_{0}^{\infty} x^{q(\lambda-1)-1} y^{q}(x) d x}{\left[\int_{0}^{\infty} x^{p \lambda-1} f^{p}(x) d x\right]^{\frac{q}{p}}}=k(p, q, \lambda),
$$

where $y(x), z(x)$ is determined by $(6.1)$. 
The values of $x_{1}, x_{2}$ are determined by (6.2). As we known in Sect. $6, y(x), z(x)$ must satisfy the following ordinary differential system:

$$
\left\{\begin{array}{l}
y^{\prime}=\frac{p(1-\lambda)}{p-1} \frac{u y}{x} \\
z^{\prime}=-x^{p \lambda-1}\left[\frac{p(1-\lambda)}{p-1} \frac{u y}{x}\right]^{p}, \quad x \in\left(x_{1}, x_{2}\right)
\end{array}\right.
$$

We define a vector valued function

$$
V(x, y, z)=\left(\begin{array}{c}
\frac{p(1-\lambda)}{p-1} \frac{u y}{x} \\
-x^{p \lambda-1}\left[\frac{p(1-\lambda)}{p-1} \frac{u y}{x}\right]^{p}
\end{array}\right), \quad(x, y, z) \in K
$$

then $V(x, y, z)$ is continuous differentiable. Therefore, $V(x, y, z)$ must satisfy the local Lipschitz condition with respect to $(y, z)$.

We point out that for any $\left(x_{0}, y_{0}, z_{0}\right) \in K$, there must be a unique function group as follow:

$$
\left\{\begin{array}{l}
y(x)=\frac{c x^{\frac{p(1-\lambda)}{p-1}}}{\left[d x^{\frac{(q-p)(1-\lambda)}{p-1}}+1\right]^{\frac{p}{q-p}}} \\
z(x)=\left[\frac{p(1-\lambda)}{p-1}\right]^{p} \int_{x}^{\infty} \frac{c^{p} t^{\frac{p(1-\lambda)}{p-1}-1}}{\left[d t^{\frac{(q-p)(1-\lambda)}{p-1}}+1\right]^{\frac{p q}{q-p}}} d t,
\end{array} \quad x \in[0, \infty),\right.
$$

such that $y\left(x_{0}\right)=y_{0}, z\left(x_{0}\right)=z_{0}$, where $c, d>0$.

In fact, for any $\left(x_{0}, y_{0}, z_{0}\right) \in K$, we set $c=\frac{y_{0}}{x_{0}^{\frac{p(1-\lambda)}{p-1}} u_{0}^{\frac{p}{q-p}}}, d=\frac{1}{x_{0}^{\frac{(q-p)(1-\lambda)}{p-1}}} \frac{1-u_{0}}{u_{0}}$, where $u_{0}=$ $u\left(x_{0}, y_{0}, z_{0}\right)$, then $c>0, d>0$, and the function group (7.1) satisfies $y\left(x_{0}\right)=y_{0}, z\left(x_{0}\right)=z_{0}$.

On the other hand, we assume that the function group (7.1) satisfy $y\left(x_{0}\right)=y_{0}$, $z\left(x_{0}\right)=z_{0}$. According to $(4.8), u(x, y(x), z(x))=\frac{1}{d x \frac{(q-p)(1-\lambda)}{p-1}+1}$. Now we denote $u_{0}=$ $u\left(x_{0}, y_{0}, z_{0}\right)$, then $\frac{1}{d x_{0}^{\frac{(q-p)(1-\lambda)}{p-1}}+1}=u_{0}$, so $c=\frac{y_{0}}{x_{0}^{\frac{p(1-\lambda)}{p-1}} u_{0}^{\frac{p}{q-p}}}, d=\frac{1}{x_{0}^{\frac{(q-p)(1-\lambda)}{p-1}}} \frac{1-u_{0}}{u_{0}}$. Therefore, the function group (7.1) is unique.

Take any $x_{0} \in\left(x_{1}, x_{2}\right)$, denote $y_{0}=y\left(x_{0}\right), z_{0}=z\left(x_{0}\right)$, then $\left(x_{0}, y_{0}, z_{0}\right) \in K$. Therefore, there is a neighborhood $\left(x_{0}-\delta, x_{0}+\delta\right) \subset\left(x_{1}, x_{2}\right)$ of $x_{0}$, such that in this region, Cauchy problem

$$
\begin{cases}y^{\prime}=\frac{p(1-\lambda)}{p-1} \frac{u y}{x}, & x \in\left(x_{1}, x_{2}\right) \\ z^{\prime}=-x^{p \lambda-1}\left[\frac{p(1-\lambda)}{p-1} \frac{u y}{x}\right]^{p}, & x \in\left(x_{1}, x_{2}\right) \\ y\left(x_{0}\right)=y_{0}, \quad z\left(x_{0}\right)=z_{0} & \end{cases}
$$

has a unique solution.

On the other hand, according to (4.9), function group (7.1) is indeed a solution of Cauchy problem (7.2), where $c=\frac{y_{0}}{x_{0}^{\frac{p(1-\lambda)}{p-1}} \frac{p}{q-p}}, d=\frac{1}{x_{0}^{\frac{(q-p)(1-\lambda)}{p-1}}} \frac{1-u_{0}}{u_{0}}, u_{0}=u\left(x_{0}, y_{0}, z_{0}\right)$. Then (7.1) holds in $\left(x_{0}-\delta, x_{0}+\delta\right)$. 
Suppose that $(a, b) \subset\left(x_{1}, x_{2}\right)$ is the greatest open interval such that (7.1) holds in $(a, b)$, then

$$
\left\{\begin{array}{l}
y(a)=\frac{c a^{\frac{p(1-\lambda)}{p-1}}}{\left[d a \frac{(q-p)(1-\lambda)}{p-1}+1\right]^{\frac{p}{q-p}}} \\
z(a)=\left[\frac{p(1-\lambda)}{p-1}\right]^{p} \int_{a}^{\infty} \frac{c^{p} t^{\frac{p(1-\lambda)}{p-1}-1}}{\left[d t^{\frac{(q-p)(1-\lambda)}{p-1}}+1\right]^{\frac{p q}{q-p}}} d t .
\end{array}\right.
$$

Set

$$
y_{a}=\frac{c a^{\frac{p(1-\lambda)}{p-1}}}{\left[d a^{\frac{(q-p)(1-\lambda)}{p-1}}+1\right]^{\frac{p}{q-p}}}, \quad z_{a}=\left[\frac{p(1-\lambda)}{p-1}\right]^{p} \int_{a}^{\infty} \frac{c^{p} t^{\frac{p(1-\lambda)}{p-1}-1}}{\left[d t^{\frac{(q-p)(1-\lambda)}{p-1}}+1\right]^{\frac{p q}{q-p}}} d t
$$

If $a>x_{1}$, there is a neighborhood $\left(a-\delta_{1}, a+\delta_{1}\right) \subset\left(x_{1}, x_{2}\right)$ of $a$, such that in this region, the Cauchy problem

$$
\begin{cases}y^{\prime}=\frac{p(1-\lambda)}{p-1} \frac{u y}{x}, & x \in\left(x_{1}, x_{2}\right) \\ z^{\prime}=-x^{p \lambda-1}\left[\frac{p(1-\lambda)}{p-1} \frac{u y}{x}\right]^{p}, & x \in\left(x_{1}, x_{2}\right) \\ y(a)=y_{a}, \quad z(a)=z_{a} & \end{cases}
$$

has a unique solution. On the other hand, function group (7.1) is indeed a solution of the Cauchy problem (7.3). Then (7.1) still holds in $\left(a-\delta_{1}, a+\delta_{1}\right)$, which is in contradiction with the choice of $(a, b)$, thus $a=x_{1}$.

Similarly, we can get $b=x_{2}$. Therefore,

$$
\left\{\begin{array}{l}
y(x)=\frac{c x^{\frac{p(1-\lambda)}{p-1}}}{\left[d x^{\frac{(q-p)(1-\lambda)}{p-1}}+1\right]^{\frac{p}{q-p}}} \\
z(x)=\left[\frac{p(1-\lambda)}{p-1}\right]^{p} \int_{x}^{\infty} \frac{c^{p} t^{\frac{p(1-\lambda)}{p-1}-1}}{\left[d t^{\frac{(q-p)(1-\lambda)}{p-1}}+1\right]^{\frac{p q}{q-p}}} d t,
\end{array} \quad x \in\left[x_{1}, x_{2}\right] .\right.
$$

Since $y\left(x_{1}\right)=0, z\left(x_{2}\right)=0$, we have $x_{1}=0$ and $x_{2}=\infty$.

On the other hand, when $y(x)=\frac{c x^{\frac{p(1-\lambda)}{p-1}}}{\left[d x \frac{(q-p)(1-\lambda)}{p-1}+1\right]}+x \in[0, \infty)(c, d>0)$, we have

$$
f(x)=y^{\prime}(x)=\frac{p(1-\lambda)}{p-1} c \frac{x^{\frac{p(1-\lambda)}{p-1}-1}}{\left[d x^{\frac{(q-p)(1-\lambda)}{p-1}}+1\right]^{\frac{q}{q-p}}}, \quad x \in(0, \infty),
$$

the equality $J(f)=k(p, q, \lambda)$ actually holds.

\section{The Proof of the Inequality Under Measurable Condition and the Determination of the Equality Condition}

Suppose that $f$ is non-negative and measurable in $(0, \infty)$ and satisfies $\int_{0}^{\infty} x^{p \lambda-1} f^{p}(x) d x=1$. $y(x), z(x)$ are still determined by (6.1). Then $y(x), z(x)$ are all absolutely continuous func- 
tions in $(0, \infty)$ and satisfy

$$
\left\{\begin{array}{l}
y^{\prime}(x)=f(x) \\
z^{\prime}(x)=-x^{p \lambda-1} f^{p}(x)
\end{array}\right.
$$

almost everywhere.

The values of $x_{1}, x_{2}$ are still determined by (6.2). Set $W(x)=W(x, y(x), z(x)), x \in$ $\left(x_{1}, x_{2}\right)$, then the equality $\lim _{x \rightarrow x_{1}^{+}} W(x)=-k(p, q, \lambda)$ still holds, and $W^{\prime}(x)=\frac{y^{q}}{x^{q(1-\lambda)+1}}+$ $\Psi(x)$ holds almost everywhere in $\left(x_{1}, x_{2}\right)$, where $\Psi(x)$ is determined by (6.3).

Take any $x_{1}<x_{3}<x_{4}<x_{2}$, then $y(x), z(x)$ are all positive and continuous functions on $\left[x_{3}, x_{4}\right]$. Suppose that the range of $\left[\frac{p-1}{p(1-\lambda)}\right]^{p} x^{p(1-\lambda)} y^{-p}(x) z(x)$ on $\left[x_{3}, x_{4}\right]$ is $[m, M]$, then $\phi^{-1}$ satisfies the Lipschitz condition on $[m, M]$ and $u=\phi^{-1}\left(\left[\frac{p-1}{p(1-\lambda)}\right]^{p} x^{p(1-\lambda)} y^{-p}(x) z(x)\right)$ is absolutely continuous on $\left[x_{3}, x_{4}\right]$. In addition, $W(x)$ is absolutely continuous on $\left[x_{3}, x_{4}\right]$, then $W\left(x_{4}\right)-W\left(x_{3}\right)=\int_{x_{3}}^{x_{4}} W^{\prime}(x) d x$.

If $x_{2}<\infty$, the equality $\lim _{x \rightarrow x_{2}^{-}} W(x)=-\frac{1}{q(1-\lambda)} \frac{y_{2}^{q}}{x_{2}^{q(1-\lambda)}}$ still holds, where $y_{2}=y\left(x_{2}\right)$. Therefore, we have

$$
\begin{aligned}
\int_{x_{1}}^{x_{2}} W^{\prime}(x) d x & =\lim _{\substack{x_{3} \rightarrow x_{1}^{+} \\
x_{4} \rightarrow x_{2}^{-}}} \int_{x_{3}}^{x_{4}} W^{\prime}(x) d x=\lim _{\substack{x_{3} \rightarrow x_{1}^{+} \\
x_{4} \rightarrow x_{2}^{-}}}\left[W\left(x_{4}\right)-W\left(x_{3}\right)\right] \\
& =k(p, q, \lambda)-\frac{1}{q(1-\lambda)} \frac{y_{2}^{q}}{x_{2}^{q(1-\lambda)}},
\end{aligned}
$$

then

$$
\begin{aligned}
\int_{0}^{\infty} x^{q(\lambda-1)-1} y^{q}(x) d x & =\int_{x_{1}}^{x_{2}} x^{q(\lambda-1)-1} y^{q}(x) d x+\int_{x_{2}}^{\infty} x^{q(\lambda-1)-1} y^{q}(x) d x \\
& \leq \int_{x_{1}}^{x_{2}} W^{\prime}(x) d x+\frac{1}{q(1-\lambda)} \frac{y_{2}^{q}}{x_{2}^{q(1-\lambda)}} \\
& =k(p, q, \lambda)-\frac{1}{q(1-\lambda)} \frac{y_{2}^{q}}{x_{2}^{q(1-\lambda)}}+\frac{1}{q(1-\lambda)} \frac{y_{2}^{q}}{x_{2}^{q(1-\lambda)}} \\
& =k(p, q, \lambda) .
\end{aligned}
$$

If and only if $x^{q(\lambda-1)-1} y^{q}(x)=W^{\prime}(x)$, namely $f=\frac{p(1-\lambda)}{p-1} \frac{u y}{x}$ holds almost everywhere in $\left(x_{1}, x_{2}\right)$, the equality holds.

Now, let's assume $x_{2}=\infty$. The inequality $W^{\prime}(x)>0$ still holds almost everywhere in $\left(x_{1}, \infty\right)$. For any $A>x_{1}$, we have

$$
\int_{x_{1}}^{A} W^{\prime}(x) d x=W(A)+k(p, q, \lambda)<k(p, q, \lambda),
$$

then $\int_{x_{1}}^{\infty} W^{\prime}(x) d x$ converges and $\int_{x_{1}}^{\infty} W^{\prime}(x) d x \leq k(p, q, \lambda)$. Therefore,

$$
\int_{0}^{\infty} x^{q(\lambda-1)-1} y^{q}(x) d x=\int_{x_{1}}^{\infty} x^{q(\lambda-1)-1} y^{q}(x) d x \leq \int_{x_{1}}^{\infty} W^{\prime}(x) d x \leq k(p, q, \lambda) .
$$


If the equality holds, then $x^{q(\lambda-1)-1} y^{q}(x)=W^{\prime}(x)$, namely $f=\frac{p(1-\lambda)}{p-1} \frac{u y}{x}$ holds almost everywhere in $\left(x_{1}, \infty\right)$.

In summary, the inequality $\int_{0}^{\infty} x^{q(\lambda-1)-1} y^{q}(x) d x \leq k(p, q, \lambda)$ always holds and if the equality holds, $f=\frac{p(1-\lambda)}{p-1} \frac{u y}{x}$ holds almost everywhere in $\left(x_{1}, x_{2}\right)$.

If $f=\frac{p(1-\lambda)}{p-1} \frac{u y}{x}$ holds almost everywhere in $\left(x_{1}, x_{2}\right)$, then for any $x \in\left(x_{1}, x_{2}\right)$, we have

$$
\left\{\begin{aligned}
y(x) & =\int_{0}^{x} f(t) d t=\int_{0}^{x_{1}} f(t) d t+\int_{x_{1}}^{x} f(t) d t=y\left(x_{1}\right)+\int_{x_{1}}^{x} \frac{p(1-\lambda)}{p-1} \frac{u y(t)}{t} d t \\
z(x) & =\int_{x}^{\infty} t^{p \lambda-1} f^{p}(t) d t=\int_{x_{1}}^{\infty} t^{p \lambda-1} f^{p}(t) d t+\int_{x}^{x_{1}} t^{p \lambda-1} f^{p}(t) d t \\
& =z\left(x_{1}\right)+\int_{x}^{x_{1}} t^{p \lambda-1}\left[\frac{p(1-\lambda)}{p-1} \frac{u y(t)}{t}\right]^{p} d t .
\end{aligned}\right.
$$

$y(x), z(x)$ are continuous in $(0, \infty)$, then $y(x), z(x)$ still satisfy the following ordinary differential system

$$
\left\{\begin{array}{l}
y^{\prime}(x)=\frac{p(1-\lambda)}{p-1} \frac{u y(x)}{x} \\
z^{\prime}(x)=-x^{p \lambda-1}\left[\frac{p(1-\lambda)}{p-1} \frac{u y(x)}{x}\right]^{p}, \quad x \in\left(x_{1}, x_{2}\right) .
\end{array}\right.
$$

According to the corresponding conclusion of Sect. 7, $x_{1}=0, x_{2}=\infty$ and there exists two positive constants $c, d$, such that

$$
\left\{\begin{array}{l}
y(x)=\frac{c x^{\frac{p(1-\lambda)}{p-1}}}{\left[d x^{\frac{(q-p)(1-\lambda)}{p-1}}+1\right]^{\frac{p}{q-p}}} \\
z(x)=\left[\frac{p(1-\lambda)}{p-1}\right]^{p} \int_{x}^{\infty} \frac{c^{p} x \frac{p(1-\lambda)}{p-1}-1}{\left[d x^{\frac{(q-p)(1-\lambda)}{p-1}}+1\right]^{\frac{p q}{q-p}}} d t,
\end{array} \quad x \in[0, \infty) .\right.
$$

On the other hand, if there exists two positive constants $c, d$, such that $y(x)=$ $\frac{c x^{\frac{p(1-\lambda)}{p-1}}}{\frac{(q-p)(1-\lambda)}{p-1}} \frac{p}{q-p}$, then $f(x)=y^{\prime}(x)=\frac{p(1-\lambda)}{p-1} c \frac{x^{\frac{p(1-\lambda)}{p-1}-1}}{\left.\frac{(q-p)(1-\lambda)}{p-1}+1\right]} \frac{q}{q-p}$, a.e. $x \in(0, \infty)$ and the equality $\int_{0}^{\infty} x^{q(\lambda-1)-1} y^{q}(x) d x=k(p, q, \lambda)$ indeed holds.

\section{Summary}

When $\lambda<1, C(p, q, \lambda)=k(p, q, \lambda)$. For any $f \in E(\lambda), J(f)=C(p, q, \lambda)$ if and only if there exists two positive constants $c, d$, such that $f(x)=\frac{c x^{\frac{p(1-\lambda)}{p-1}-1}}{\left[d x^{\frac{(q-p)(1-\lambda)}{p-1}}+1\right]} \frac{q}{q-p}$, a.e. $x \in(0, \infty)$.

When $\lambda>1$, by Lemma 2.2 and the symmetry of $k(p, q, \lambda)$, we have

$$
C(p, q, \lambda)=C(p, q, 2-\lambda)=k(p, q, 2-\lambda)=k(p, q, \lambda) .
$$

Still based on Lemma 2.2, for any $f \in E(\lambda), J(f)=C(p, q, \lambda)$ if and only if

$$
J\left(\frac{1}{x^{2}} f\left(\frac{1}{x}\right)\right)=C(p, q, 2-\lambda) .
$$

The equality (9.1) holds if and only if there exists two positive constants $c, d$, such that

$$
\frac{1}{x^{2}} f\left(\frac{1}{x}\right)=\frac{c x^{\frac{p(1-\lambda)}{p-1}-1}}{\left[d x^{\frac{(q-p)(1-\lambda)}{p-1}}+1\right]^{\frac{q}{q-p}}}, \quad \text { a.e. } x \in(0, \infty)
$$


that is

$$
f(x)=\frac{c x^{\frac{(q-p)(\lambda-1)}{p-1}-1}}{\left[d+x^{\frac{(q-p)(\lambda-1)}{p-1}}\right]^{\frac{q}{q-p}}}, \quad \text { a.e. } x \in(0, \infty) .
$$

Therefore, $J(f)=C(p, q, \lambda)$ if and only if there exists two positive constants $c, d$, such that

$$
f(x)=\frac{c x^{\frac{(q-p)(\lambda-1)}{p-1}-1}}{\left[d x^{\frac{(q-p)(\lambda-1)}{p-1}}+1\right]^{\frac{q}{q-p}}}, \quad \text { a.e. } x \in(0, \infty) .
$$

Till now, we have proved Theorem 1.5 completely.

Publisher's Note Springer Nature remains neutral with regard to jurisdictional claims in published maps and institutional affiliations.

Open Access This article is licensed under a Creative Commons Attribution 4.0 International License, which permits use, sharing, adaptation, distribution and reproduction in any medium or format, as long as you give appropriate credit to the original author(s) and the source, provide a link to the Creative Commons licence, and indicate if changes were made. The images or other third party material in this article are included in the article's Creative Commons licence, unless indicated otherwise in a credit line to the material. If material is not included in the article's Creative Commons licence and your intended use is not permitted by statutory regulation or exceeds the permitted use, you will need to obtain permission directly from the copyright holder. To view a copy of this licence, visit http://creativecommons.org/licenses/by/4.0/.

\section{References}

1. Hardy, G.H., Littlewood, J.E.: Notes on the theory of series (XII): on certain inequalities connected with the calculus of variations. J. Lond. Math. Soc. 5, 283-290 (1930)

2. Bliss, G.A.: An integral inequality. J. Lond. Math. Soc. 5, 40-46 (1930)

3. Flett, T.M.: A note on some inequalities. Glasg. Math. J. 4(1), 7-15 (1958)

4. George, C.: Exercise in Integration. Springer, Berlin (1984)

5. Bradley, J.S.: Hardy inequality with mixed norms. Can. Math. Bull. 21(3), 405-408 (1978)

6. Muckenhoupt, B.: Hardy's inequality with weights. Stud. Math. 34(2), 31-38 (1972)

7. Beesack, P.R., Heinig, H.P.: Hardy's inequality with indices less than 1. Proc. Am. Math. Soc. 83(2), 532-536 (1981)

8. Li, Y., Mao, Y.: The optimal constant in generalized Hardy's inequality. Math. Inequal. Appl. 23(1), 257-266 (2020)

9. Love, E.R.: Generalizations of a classical inequality. Appl. Anal. 8(1), 47-59 (1978)

10. Andersen, K.F., Muckenhoupt, B.: Weighted weak type Hardy inequalities with applications to Hilbert transforms and maximal functions. Stud. Math. 72(1), 9-26 (1982)

11. Heinig, H.P., Stepanov, V.D.: Weighted Hardy inequalities for increasing functions. Can. J. Math. 45(1), 104-116 (1993)

12. Bergh, J., Burenkov, V., Persson, L.E.: On some sharp reversed Holder and Hardy type inequalities. Math. Nachr. 169(1), 19-29 (1994)

13. Cizmesija, A., Krulict, K., Pecaric, J.: A new class of general refined Hardy-type inequalities with kernels. Math. Inequal. Appl. 4(4), 481-503 (2013)

14. Chen, M.: The optimal constant in Hardy-type inequalities. Acta Math. Sin. 31(5), 731-754 (2015)

15. Mitrinovic, D.S., Pecaric, J.E., Fink, A.M.: Inequalities Involving Functions and Their Integrals and Derivatives. Kluwer Academic, Dordrecht (1991)

16. Kuang, J.: Applied Inequalities. Shandong Science and Technology Press, Jinan (2010)

17. Apostol, T.M.: Mathematical Analysis. China Machine Press, Beijing (2004) 